\section{Mortalidade materna e sua interface com a raça em Mato Grosso}

\section{Maternal mortality and race in the Brazilian State of Mato Grosso}

\section{Abstract}

Objectives: to examine the correlation between maternal mortality rates and race/color in the Brazilian State of Mato Grosso between 2000 and 2006.

Methods: an epidemiological study was carried out using the death certificates, DATASUS and SIM/SES-MT to provide a time series for these years. The race/color of the women who died during childbirth was related to the variables age, level of education, marital status, primary cause of death and type of obstetric cause. In combination with the $\chi^{2}$ test and the odds rati were used.

Results: the $\chi^{2}$ test showed a significant association between race/color and maternal mortality, with a level of significance of $95 \%$. The odds ratio revealed that such deaths were 5.13 times more likely among black women and 5.71 times more likely among indigenous women, compared to white women. "Hypertensive disorders during pregnancy, childbirth or puerperium" were the main cause of maternal mortality among African Brazilian women and women of mixed race; $45.4 \%$ and $29.93 \%$ respectively. Indigenous women died more of "complications during labor" with $27.2 \%$. White women died more (30.7\%) of "other obstetric complications".

Conclusions: in the state of Mato Grosso a high maternal mortality rate from direct obstetric causes are found among black and indigenous women.

Key words Maternal mortality, Ethnic group and health, Vital statistics, Information systems
Neuma Zamariano Fanaia Teixeira 1

Wilza Rocha Pereira 2

Dulce Aparecida Barbosa 3

Lucila Amaral Carneiro Vianna 4

1,2 Faculdade de Enfermagem. Universidade Federal de Mato Grosso. Av. Fernando Corrêa da Costa, 2367. Bairro Boa Esperança. Cuiabá, MT, Brasil. CEP: 78.060-900. E-mail: neuma.zamariano@gmail.com 3,4 Departamento de Enfermagem. Universidade Federal de São Paulo. São Paulo, SP, Brasil

\section{Resumo}

Objetivos: analisar a correlação entre óbitos maternos e raça/cor no Estado de Mato Grosso entre os anos de 2000 a 2006.

Métodos: estudo epidemiológico com base nos dados da Declaração de Óbitos, DATASUS e SIM/SES-MT, que propiciaram a análise da série histórica entre esses anos. Relacionou-se raça/cor das mulheres que foram a óbito por causas maternas com as variáveis idade, escolaridade, estado civil, causa básica de óbito e tipo de causa obstétrica. Utilizaram-se teste de $\chi^{2}$ e odds ratio.

Resultados: o teste $\chi^{2}$ evidenciou associação significativa entre raça/cor e morte materna, ao nível de significância de $95 \%$. A odds ratio apontou uma razão de chances dessas mortes 5,13 vezes maior para mulheres pretas e 5,71 para indigenas, comparadas às mulheres brancas. "Transtornos hipertensivos da gravidez, parto/puerpério" foram a principal causa de óbito materno para as afrodescendentes e pardas; respectivamente $45,4 \%$ e $29,93 \%$. As indigenas morreram mais pelas "complicações do trabalho de parto/parto" com 27,2\%. Das mulheres brancas, 30,7\% morreram por "outras complicações obstétricas não classificadas em outras causas".

Conclusões: em todo Mato Grosso as mortes maternas por causas obstétricas diretas pervaleceu entre mulheres pretas e indígenas.

Palavras-chave Mortalidade materna, Etnia e saúde, Estatísticas vitais, Sistemas de informação 


\section{Introdução}

A gravidez é vista pela maioria das mulheres como um momento especial, pois o nascimento de um membro da família significa a continuidade da vida e a renovação de um núcleo familiar. Porém, existem casos em que a gravidez acarreta riscos para a mãe e o feto, sendo denominada de gestação de alto risco, ${ }^{1}$ ou seja, aquela na qual a vida ou saúde da mãe, feto ou recém-nascido, pode ser prejudicada por intercorrências diversas. Há fatos intrínsecos associados à concepção, gestação e parto, e às questões relacionadas às práticas em saúde, que podem fazer desse momento um evento a ser comemorado ou lastimado neste caso quando ocorre a morte materna (MM).

Considera-se morte materna a morte de uma mulher durante a gestação ou até 42 dias após o término desta, independentemente de sua duração, sendo causada por fatores relacionados ou agravados pela gravidez ou por medidas tomadas em relação à ela. ${ }^{2}$

A razão de mortalidade materna (RMM) referese às mortes maternas obstétricas diretas e indiretas com o número de nascidos vivos (NV), e é expressa por $100.000 \mathrm{NV} .{ }^{2}$ Esta difere significativamente entre nações desenvolvidas e em desenvolvimento. Enquanto Canadá e Estados Unidos apresentaram razões inferiores a nove óbitos por $100.000 \mathrm{NV}$, o Brasil 3 atingiu 260 óbitos/ $100.000 \mathrm{NV}$ em 2000. Entretanto, em alguns países em desenvolvimento nos quais as políticas de saúde são mais elaboradas, como Cuba e Costa Rica, essas taxas são inferiores a 40 por $100.000 \mathrm{NV}, 2$ um valor ainda considerado elevado quando se trata da vida humana.

A World Health Organization (WHO) revelou que, em 2005, morreram em todo o mundo 536.000 mulheres por causas relacionadas à gestação, parto $\mathrm{e}$ puerpério, com uma taxa de $99 \%$ destas mortes ocorridas nos países em desenvolvimento. ${ }^{4} \mathrm{~A}$ WHO realizou análises de tendência de $\mathrm{MM}$ e constatou seu decréscimo anual, em média menor que $1 \%$ entre 1990 e 2005. O quinto objetivo de desenvolvimento do milênio definiu, em 2000, uma taxa desejada de declínio da $\mathrm{MM}$ de 5,5\% anualmente, ${ }^{4}$ a qual não foi alcançada.

Esses números, bem conhecidos e divulgados, representam o onus global das mortes relacionadas com a gravidez, convidando à reflexão sobre o que pode ser feito em curto prazo. Portanto, a problematização dessas mortes tornou-se objeto de uma vigilância cuidadosa em vários países, exigindo assim, uma preocupação cada vez maior no sentido de se conhecerem todas as mortes neles ocorridas. 5
No Brasil a estimativa de mortes maternas para todos os países torna-se a base para o planejamento de programas de saúde sexual e reprodutiva, além de um guia aos esforços e pesquisas para melhorar a saúde materna, comparando-a aos índices encontrados nos países desenvolvidos. ${ }^{4}$

Mulheres pretas e pardas encontram-se entre os grupos mais vulneráveis ao óbito materno; embora um grupo social não se defina por relações de raça ou cor, diferenças étnicas associam-se a desigualdades sociais e condicionam a forma de viver de grupos e pessoas. A etnia não é por si só fator de risco, mas a inserção social adversa do grupo racial constitui-se em vulnerabilidade. $6-7$

São escassos em nosso país os dados de MM com recorte racial/étnico, porém eles são necessários por extrapolarem os aspectos meramente biológicos do processo saúde-doença. Um estudo sobre aspectos epidemiológicos relacionados aos fatores étnico-raciais no Brasil mostrou que os piores indicadores de mortalidade em termos de causas evitáveis, a exemplo da MM, são relativos às mulheres afrodescendentes e indígenas, 8 evidenciando a relação entre a raça e a desassistência à saúde de alguns grupos sociais.

Há um potencial patogênico pouco visível nas discriminações de qualquer ordem e isso contribui para melhor compreender a amplitude do conceito de vulnerabilidade e o avanço que ele representa quando se contrapõe ao conceito de risco. ${ }^{6}$ As mulheres afrodescendentes e indígenas estão na intersecção das discriminações de raça, de gênero e de classe social e estão expostas a um risco aumentado de compro-meter a sua autorreferência pessoal, imagem cor-poral, autoconceito e autoestima. Assim, as ações de saúde de combate à discriminação 8 e à exclusão da mulher afrodescendente deve ser desenvolvida integralmente, considerando o corpo, as relações de gênero e também as relações políticas, ou seja, aquelas que convidam à emancipação pelo empoderamento feminino.

Neste contexto o presente artigo objetivou analisar a relação entre os óbitos maternos e raça/cor no Estado de Mato Grosso entre os anos de 2000 e 2006, relacionando-os com idade, escolaridade, estado civil, causa básica de óbito e tipo de causa obstétrica das 219 mulheres que foram a óbito no período estudado.

\section{Métodos}

Estudo epidemiológico descritivo que teve como base os dados da Declaração de Óbitos (DO) por causas maternas que propiciou análise da série 
histórica no período de 2000 a 2006 no Estado do Mato Grosso. Foram obtidos registros de 219 óbitos maternos ocorridos no Estado de Mato Grosso, a partir do Departamento de Informática do Sistema Único de Saúde (DATASUS) do Ministério da Saúde (MS), bem como dados do Sistema de Informação em Mortalidade da Secretaria Estadual de Saúde do Estado Mato Grosso (SIM/SES-MT). Estes óbitos foram analisados utilizando-se odds ratio (OR) e tabelas de contingência, a partir das quais se relacionou a raça/cor com as variáveis idade, escolaridade, estado civil, causa básica do óbito e tipo de causa obstétrica, bem como gráficos indicando séries históricas dos óbitos maternos no período dos sete anos estudados.

Caracterizou-se a raça/cor segundo a classificação do Instituto Brasileiro de Geografia e Estatística (IBGE) como: branca, preta, parda, amarela e indígena. A categoria de raça/cor amarela não foi incluída no estudo, pois no período do mesmo não houve óbito nesta população.

As seguintes variáveis foram utilizadas seguindo classificação do DATASUS: idade (faixas etárias de dez anos), escolaridade (nenhuma, ensino fundamental, ensino médio e superior, ignorada), estado civil (solteira, casada, viúva, separada judicialmente, outro/ignorada), causa básica (capítulo XV da Classificação Internacional das Doenças $10^{\mathrm{a}}$ revisão - CID 10 que classifica as doenças do ciclo gravídico-puerperal) e tipo de causa obstétrica (morte materna obstétrica direta e indireta).

Foi calculada a RMM por raça/cor no período do estudo, bem como a OR para as 219 mulheres. Para o cálculo da OR, considerou-se como não acometidos os nascidos vivos (segundo a raça/cor) a partir dos dados do Sistema de Informações sobre Nascidos Vivos (SINASC/DATASUS), no entanto, o resultado configurou-se apenas como estimativa, pois algumas das mães que foram a óbito tiveram natimortos e gemelares que não estão computados nos dados. Usou-se, como comparação para a OR, a raça branca. Pretendeu-se, assim, melhor visualizar se a distribuição destes óbitos segundo a raça/cor era aleatória ou se havia um padrão determinado de dependência dessas variáveis. Utilizou-se o teste $\chi^{2}$ para avaliar associação entre as mortes maternas e raça/cor. Adotou-se um intervalo de confiança de $95 \%$ e um nível $p<0,05$ para evidenciar a significância estatística entre raça/cor e óbitos maternos. Os cálculos foi efetuados no Programa EPI-INFO 6.0, utilizando-se o Statcalc.

O estudo teve início após aprovação do Comitê de Ética em Pesquisa sob número 894/CEPUNIFESP/HSP/08; todas as orientações da
Resolução CNS 196/96 foram seguidas.

\section{Resultados}

Das 219 mulheres analisadas, 58,0\% pertenciam à raça/cor parda; $29,7 \%$ à branca, $5,0 \%$ à preta e, $5,0 \%$ à indígena. $\mathrm{O}$ dado raça/cor não foi registrado na DO de cinco mulheres $(2,3 \%)$ (Tabela 1$)$.

As maiores concentrações de óbitos ocorreram no grupo de 20 a 29 anos entre as mulheres pardas $(52,8 \%)$; e entre as brancas e pretas $36,9 \%$. No entanto, a maior concentração de óbitos entre as indígenas $(36,4 \%)$ foi na faixa etária de 30 a 39 anos (Tabela 1).

Considerando-se o estado civil desta população, morreram principalmente as mulheres solteiras pretas, pardas e indígenas, respectivamente, $72,7 \%$, $58,3 \%$ e $36,4 \%$. Quando se considera o estado civil e a cor verifica-se que as mulheres brancas e casadas apresentaram a maior concentração de óbitos maternos (40,0\%). Vale destacar que, em $36,4 \%$ dos registros de óbitos, não constava o estado civil, o que prejudica a análise da variável.

Identificou-se, quanto à escolaridade, que 56,7\% e $44,6 \%$ das mulheres pardas e brancas, respectivamente, cursaram o ensino fundamental; no entanto, entre as pretas, o percentual foi de $36,4 \%$ igualmente para o ensino fundamental e para o ensino médio.

A principal causa de óbito materno foram outras complicações obstétricas não classificadas em outras causas (NCOP), perfazendo 30,7\% das mulheres brancas. Para as pretas e pardas foram: edema, proteinúria, transtornos hipertensivos da gravidez, parto e puerpério com $45,4 \%$ e $29,9 \%$, respectivamente; para as indígenas, a causa principal foram complicações do trabalho de parto e parto com 27,2\% (Tabela 2).

Analisando a evolução da RMM (Figura 1) referente às causas obstétricas diretas, a partir da série histórica, observou-se elevação com posterior queda no ano de 2004 para as mulheres pretas, atingindo, em 2006, uma RMM de 392,9 óbitos/100.000 NV. Considerando-se as indígenas, houve o topo em 2001 com RMM de 456,2 óbitos/100.000 NV. Para as brancas, a RMM alcançou seu ápice em 2000 com 44,7 óbitos/100.000 NV e, para as pardas, RMM 69,5/100.000 NV em 2005.

$\mathrm{Na}$ Figura 2, evidencia-se RMM de 347,2 óbitos/100.000 NV por causas indiretas para as indígenas em 2000, e de 141 óbitos/100.000 NV para as pretas em 2004. Para as brancas e pardas, esta razão não se elevou além de 33,5 óbitos/100.000 NV.

Segundo os dados do DATASUS em relação ao número de óbitos maternos e de nascidos vivos no 
Tabela 1

Características sociodemográficas, segundo raça/cor de mulheres que faleceram por causas maternas, Mato Grosso, 2000 a 2006

\begin{tabular}{|c|c|c|c|c|c|c|c|c|}
\hline \multirow{3}{*}{ Características* } & \multicolumn{8}{|c|}{ Raça/Cor } \\
\hline & \multicolumn{2}{|c|}{ Branca $(n=65)$} & \multicolumn{2}{|c|}{ Parda $(n=127)$} & \multicolumn{2}{|c|}{ Preta $(n=11)$} & \multicolumn{2}{|c|}{ Indígena $(n=11)$} \\
\hline & $\mathrm{n}$ & $\%$ & $\mathrm{n}$ & $\%$ & $\mathrm{n}$ & $\%$ & $\mathrm{n}$ & $\%$ \\
\hline \multicolumn{9}{|l|}{ Idade (anos) } \\
\hline 10 a 19 & 14 & 21,5 & 24 & 18,9 & 3 & 27,3 & 2 & 18,2 \\
\hline 20 a 29 & 24 & 36,9 & 67 & 52,8 & 4 & 36,4 & 3 & 27,2 \\
\hline 30 a 39 & 21 & 32,3 & 31 & 24,4 & 3 & 27,3 & 4 & 36,4 \\
\hline 40 a 49 & 6 & 9,2 & 5 & 3,9 & 1 & 9,0 & 2 & 18,2 \\
\hline \multicolumn{9}{|l|}{ Estado civil } \\
\hline Solteira** & 25 & 38,5 & 74 & 58,3 & 8 & 72,7 & 4 & 36,4 \\
\hline Casada & 26 & 40,0 & 34 & 26,8 & 3 & 27,3 & 3 & 27,2 \\
\hline Separada judicialmente & - & - & 1 & 0,8 & - & - & - & - \\
\hline Viúva & - & - & 1 & 0,8 & - & - & - & - \\
\hline Outro/lgnorado & 14 & 21,5 & 17 & 13,3 & - & - & 4 & 36,4 \\
\hline \multicolumn{9}{|l|}{ Escolaridade } \\
\hline Nenhuma & 2 & 3,1 & 5 & 3,9 & 2 & 18,1 & 4 & 36,4 \\
\hline Fundamental & 29 & 44,6 & 72 & 56,7 & 4 & 36,4 & 1 & 9,1 \\
\hline Médio & 18 & 27,7 & 27 & 21,3 & 4 & 36,4 & 1 & 9,1 \\
\hline Superior & 8 & 12,3 & 6 & 4,7 & 1 & 9,1 & - & - \\
\hline Ignorado & 8 & 12,3 & 17 & 13,4 & - & - & 5 & 45,4 \\
\hline
\end{tabular}

*Não havia informação acerca da raça/cor na declaração de óbito de cinco mulheres com relação à idade, estado civil e escolaridade; **Duas mulheres solteiras com raça ignorada.

Tabela 2

Causas de óbitos maternos segundo raça/cor, Mato Grosso, 2000 a 2006.

\begin{tabular}{|c|c|c|c|c|c|c|c|c|}
\hline \multirow{3}{*}{ Causas } & \multicolumn{8}{|c|}{ Raça/Cor } \\
\hline & \multicolumn{2}{|c|}{ Branca $(n=65)$} & \multicolumn{2}{|c|}{ Preta $(n=11)$} & \multicolumn{2}{|c|}{ Parda $(n=127)$} & \multicolumn{2}{|c|}{ Indígena $(n=11)$} \\
\hline & $\mathrm{n}$ & $\%$ & $\mathrm{n}$ & $\%$ & $\mathrm{n}$ & $\%$ & $\mathrm{n}$ & $\%$ \\
\hline Gravidez terminando em aborto & 8 & 12,3 & 1 & 9,1 & 9 & 7,1 & 1 & 9,1 \\
\hline $\begin{array}{l}\text { Edems, proteinúria, transtornos } \\
\text { hipertensivos da gravidez parto } \\
\text { e puerpério* }\end{array}$ & 15 & 23,1 & 5 & 45,4 & 38 & 29,9 & 1 & 9,1 \\
\hline $\begin{array}{l}\text { Outros transtornos maternos } \\
\text { relacionados com a gravidez }\end{array}$ & 2 & 3,1 & 1 & 9,1 & 10 & 7,9 & 2 & 18,2 \\
\hline $\begin{array}{l}\text { Assististência à mãe ligadas ao } \\
\text { feto, cavidade amniótica, proble- } \\
\text { ma de parto }\end{array}$ & 2 & 3,1 & - & - & 6 & 4,7 & 1 & 9,1 \\
\hline $\begin{array}{l}\text { Complicações do trabalho de } \\
\text { parto e parto** }\end{array}$ & 10 & 15,4 & - & - & 22 & 17,3 & 3 & 27,2 \\
\hline $\begin{array}{l}\text { Complicações relacionadas ao } \\
\text { puerpério }\end{array}$ & 6 & 9,2 & 2 & 18,2 & 17 & 13,4 & 2 & 18,2 \\
\hline
\end{tabular}

*1 caso de raça ignorada; **3 casos de raça ignorada Fonte: DATASUS 


\section{Figura 1}

Série histórica da razão de morte materna obstétrica direta segundo raça/cor, Mato Grosso, 2000 a 2006.

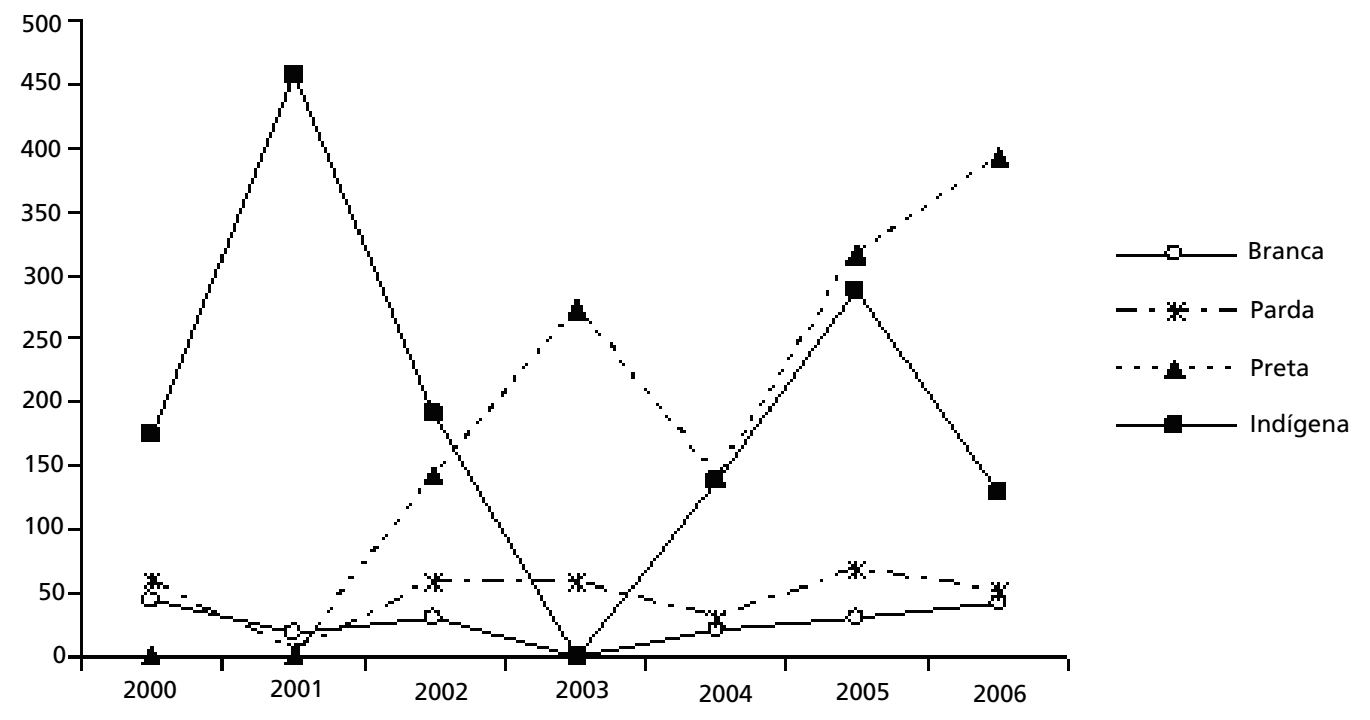

Fonte: DATASUS.

Figura 2

Série histórica da razão de morte materna obstétrica indireta segundo raça/cor, Mato Grosso, 2000 a 2006.

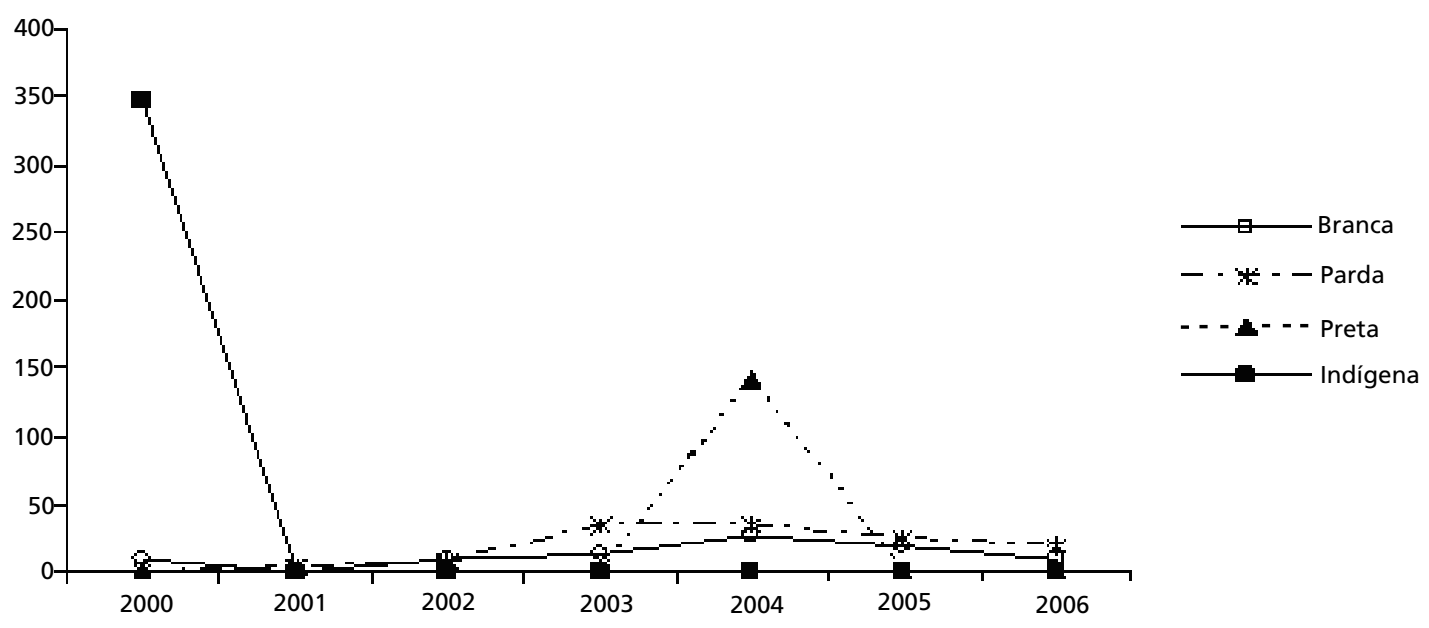

Fonte: DATASUS. 
período do estudo, a OR foi cinco vezes maior para as pretas e indígenas quando comparadas às brancas
(Tabela 3).

Tabela 3

Odds ratio de óbitos maternos segundo raça/cor, Mato Grosso, 2000 a 2006

\begin{tabular}{lcccc}
\hline Relação entre raça/Cor & Odds ratio & IC95\% & $\chi^{2}$ & $\boldsymbol{p}$ \\
\hline Preta/branca & 5,13 & $2,56-10,04$ & 31,25 & $<0,001$ \\
Parda/branca & 1,68 & $1,23-2,29$ & $11,73<0,001$ & $<, 46$ \\
Indígena/branca & 5,71 & $2,85-11,16$ & 36,46 & $<0,001$ \\
\hline
\end{tabular}

\section{Discussão}

De acordo com o Censo Brasileiro de 2000 do Instituto Brasileiro de Geografia e Estatística (IBGE), $944 \%$ da população brasileira era composta por afrodescendentes, sendo $49,1 \%$ de mulheres entre 10 a 49 anos, faixa etária considerada na RMM. Para Mato Grosso, foram 53,1\% de mulheres com essa descendência na mesma faixa etária e período.

Mulheres afrodescendentes (mulheres pretas, bem como as mestiças de indivíduos brancos e pretos) geralmente apresentam nível socioeconômico baixo; em relação à saúde reprodutiva, têm menos acesso aos métodos contraceptivos, o que proporciona mais chances de gravidez. 10 Juntamente com as mulheres indígenas, as afrodescendentes no Brasil concentraram os maiores índices de MM para as regiões Norte, Nordeste e Centro-Oeste, que inclui o Estado onde o estudo foi feito.

Os dados apontaram que $63 \%$ dos óbitos maternos ocorridos no Mato Grosso naquele ano deram-se entre as afrodescendentes, proporcionalmente $10 \%$ a mais ao se comparar com o percentual representativo desta população feminina no período considerado. A proporção de óbitos por falha na assistência médica pode ser considerada uma evidência de diferenças entre grupos étnico-raciais, ao tomarem-se por referência, condições de acesso aos serviços de saúde.

$\mathrm{Na}$ faixa etária compreendida entre 20 e 29 anos, auge da vida reprodutiva e produtiva, observou-se uma quantidade representativa de óbitos maternos para as mulheres brancas e pardas. O percentual mais elevado nas mulheres indígenas na faixa de 30 a 39 anos, pode indicar que são relegadas à orfandade um número significativo de crianças. Comparativamente, nos Estados Unidos da América (EUA), um estudo11 revelou que mulheres afrodescendentes tiveram maior taxa de MM no grupo de 35 a 45 anos.

Estudo realizado em todas as capitais brasileiras em 2002, sobre a morte de mulheres em idade fértil com ênfase na MM,12 revelou, para a região CentroOeste, 18 casos de óbitos por causas maternas pela declaração de óbito (DO) original. Após a coleta de dados que os reavaliou, esse número foi acrescido de mais dez óbitos, revelando então 28 óbitos por causas maternas. Destes, $28,6 \%$ ocorreram entre 25 a 29 anos, e $21,4 \%$ igualmente, nas faixas de 15 a 19 anos e 35 a 39 anos. No que se refere à faixa etária, percebe-se que os dados acima coincidem com aqueles levantados neste estudo.

O estado civil das mulheres do presente estudo revelou que grande parte das brancas era casada, já para as outras raças, o estado civil mais representativo foi o de solteira. Este quesito revela dificuldades avaliativas, uma vez que, dependendo de como é abordado, a resposta pode não ser representativa da realidade conjugal dessas mulheres, pois, o status de casada é mais bem considerado do que o de não casada. ${ }^{13}$ Este dado poderia justificar o fato de constatar-se um número maior de óbitos maternos entre as mulheres solteiras.

Concernente a relação entre qualidade de cuidados obstétricos e estado civil, foi evidenciado um declínio na RMM na Alemanha 14 em razão das melhorias na acessibilidade e qualidade dos cuidados obstétricos que beneficiaram igualmente mulheres solteiras e casadas. No entanto, essas melhorias não conseguiram reduzir a disparidade na $\mathrm{MM}$ entre essas duas populações, pois o aumento no risco que acometia mulheres solteiras persistiu por quase duas décadas enquanto para as casadas, foi imediato.

Nos EUA, estudo que abordou a relação entre assistência obstétrica e raça, verificou-se baixa disparidade na assistência obstétrica entre brancas e afrodescendentes. 15 Estas mantiveram maior utilização de assistência pré-natal intensiva, por terem maior risco de resultados adversos na 
gravidez, explicando a maior busca por assistência na clientela investigada. Reportando-se ao Estado de Mato Grosso na relação entre escolaridade e MM, dentre as mulheres que foram a óbito no presente estudo, a maioria cursou apenas o ensino fundamental. Uma pesquisa com mulheres americanas afrodescendentes com ensino médio incompleto ${ }^{11}$ apontou uma taxa de MM 1,5 vezes maior que as mesmas com ensino médio completo. Assim, o presente estudo vai ao encontro dessa pesquisa, ao revelar que a escola-ridade exerce significativa influência sobre a morta-lidade materna, independentemente da raça/cor.

Além de a escolaridade exercer influência na qualidade da assistência obstétrica recebida pelas mulheres, outro elemento importante remete à educação em saúde, principalmente em prol do parto normal para a redução de cesáreas desnecessárias. Não é prática corrente dos profissionais de saúde no Estado de Mato Grosso informar as mulheres dos riscos adicionais do parto operatório que podem resultar em uma MM até 12 vezes maior do que a observada quando do parto vaginal, 16 além do que a cesariana implica o dobro de tempo da permanência hospitalar, e pode gerar transtornos respiratórios neonatais e prematuridade iatrogênica.

Um estudo com mulheres de baixa renda sobre a vivência do parto17 evidenciou que, corriqueiramente, não há por parte dos profissionais a compreensão da mulher como um ser de direitos sobre seu corpo, pois prevalecem, quase sempre, os saberes profissionais sobre os saberes da experiência vivida no próprio corpo pelas mulheres no ciclo gravídico-puerperal.

A desinformação reflete na falta de percepção da gravidade da morte materna. Esta falta de percepção relaciona-se à questão cultural: para muitos, a morte materna pode acontecer até por causa divina, sendo pouco evidente tratar-se de um problema evitável e de saúde pública. 18

A ocorrência das mortes por causas maternas também revela que a relação hierárquica entre gênero e raça é ainda pouco visibilizada, havendo forte evidência de que ser mulher e afrodescendente no nosso país é indício de vulnerabilidade. ${ }^{7}$ Essa relação induz os profissionais a desapropriá-las dos direitos básicos, como o direito à vida e ao cuidado com a saúde, pois há mortes de mulheres afrodescendentes que não têm causas em doenças pré-existentes, revelando que, nesta população, estão entre as causas mais frequentes, as complicações da gravidez e parto, dentre outras.

Óbitos por doença hipertensiva da gravidez são frequentes entre as mulheres afrodescendentes, como também a taxa para estes é quase seis vezes maior do que entre mulheres brancas. Em razão de serem, em sua maioria, chefes de família sem cônjuge, mas com filhos, a MM de mulheres afrodescendentes, consequentemente relega à orfandade grande número de crianças. 19

Várias evidências sugerem que essas causas estão relacionadas à predisposição biológica das afrodescendentes para doenças como a hipertensão arterial, ${ }^{7}$ a fatores relacionados à dificuldade de acesso $^{1}$ e à baixa qualidade do atendimento, 15 bem como à falta de ações e capacitação de profissionais de saúde voltadas para atender aos riscos específicos aos quais elas estão expostas.

Uma pesquisa nos EUA que avaliou disparidades raciais relacionadas à $\mathrm{MM}$ evidenciou uma maior prevalência e/ou gravidade de complicações cardiovasculares, pré-eclampsia, hemorragia e infecção entre mulheres americanas afrodescendentes. 20

Para as mulheres brancas, a principal causa de MM comprovada pelos dados do SIM/SES-MT foram outras afecções obstétricas não computadas em outras causas, a exemplo das mortes por sequela de causas obstétricas diretas, doenças infecciosas e parasitárias classificadas em outras causas, mas agravadas pela gravidez, parto e puerpério, causas essas que não foram de todo esclarecidas.

A falta de conhecimento da real causa que leva a morte no ciclo reprodutivo dificulta o planejamento de ações que visem sua redução. O preenchimento incorreto da $\mathrm{DO}$ e o sub-registro do evento nas estatísticas oficiais são fatores que dificultam o monitoramento correto do nível da MM no Brasil. 3

Uma causa que merece destaque, a partir dos dados levantados, relaciona-se às complicações do trabalho de parto e parto que comumente levam a hemorragia e infecção. Há tempos, o conhecimento da hemorragia como uma das principais causas de mortes maternas são reveladas pela prática obstétrica advinda de profissionais não competentes. $\mathrm{O}$ óbito por hemorragia, durante ou após o parto, decorre em grande medida da falha no acesso à oportuna e competente assistência obstétrica. ${ }^{21}$

Os óbitos por hemorragia entre as mulheres americanas afrodescendentes desvelaram menor intervenção cirúrgica frente a esta intercorrência quando comparadas às brancas, 22 reafirmando a expropriação de direitos a que estão expostas essas mulheres.

A elevada RMM das causas obstétricas diretas, evidenciada na série histórica, decorreu em $100 \%$ dos casos de complicações do trabalho de parto e parto para as indígenas; para as mulheres pretas, derivou de transtornos hipertensivos de acordo com 
o SIM/SES-MT. Observou-se variações nos óbitos em números absolutos entre as raça/cor preta e indígena nos anos de 2001, 2003 e 2006, respectivamente, um, dois e dois óbitos para as mulheres pretas e três, zero e um óbito para as indígenas, evidenciando a flutuação nestes números de óbitos durante a série histórica. Dados do MS revelam que as principais causas de morte materna derivam das causas obstétricas diretas, que são ocasionadas por patologias preveníveis com uma assistência qualificada em saúde. ${ }^{3}$

A avaliação dos dados reafirmou a não melhoria da assistência a essas mulheres, contrapondo-se ao verificado em países desenvolvidos que investem nesta investigação 23 e consideram que, para entender essas taxas elevadas, são necessários uma completa interação e o desvelar entre o biológico e os fatores relativos aos serviços de saúde.

Estudando-se os óbitos obstétricos indiretos, decorrentes do agravamento de causas pré-existentes, encontrou-se um ápice para as indígenas em 2000 e para as pretas em 2004. Para as brancas e pardas, a RMM por essas causas apresentou pequenas flutuações durante os anos estudados.

Analisando-se a RMM por todas as causas, constataram-se taxas muito elevadas para as pretas nesses sete anos, chegando a 500 óbitos/100.000 NV. Para as indígenas, a RMM manteve-se acima de 100 óbitos/100.000 NV, com ápice em 2001 por ter ocorrido dois óbitos por causa direta nessa raça naquele ano; no entanto em 2003 não se registrou esta ocorrência. Para as brancas, este indicador manteve-se abaixo de 50 óbitos/100.000 NV e para as pardas, um pouco acima deste patamar. Esses dados apontaram o risco a que estão expostas ao óbito materno as mulheres pretas e indígenas residentes no Estado de Mato Grosso.

Um estudo 24 que examinou a MM de mulheres pretas evidenciou o risco adicional a que estão submetidas, pois apresentaram para todas as causas uma RMM de 77,9 óbitos/100.000 NV, enquanto que, para as brancas, foi de 38,2 óbitos/100.000 NV. Considerando o aborto como causa de MM, o estudo apontou um diferencial nestas taxas: 9,4 óbitos/100.000 NV entre as pretas e 3,2 óbitos/100.000 NV entre as brancas.

Analisando os óbitos maternos relativos à raça/cor, notou-se que o OR foi consideravelmente maior para as pretas e indígenas quando comparadas às brancas, indo ao encontro do revelado pela RMM

\section{Referências}

1. Brasil. Ministério da Saúde. Secretaria de Políticas de Saúde. Área Técnica de Saúde da Mulher. Gestação de alto dessas mulheres. Entre as pardas, o OR revelou uma vez e meia mais de chance de ocorrer o óbito na relação com as brancas. Outro estudo ${ }^{25}$ observou que o risco relativo das mulheres que foram a óbito por causas maternas foi 7,4 vezes maior nas pretas e cinco vezes maior entre as amarelas, quando comparadas às brancas. Dados muito próximos aos do presente estudo foram também encontrados para as pretas e indígenas. A taxa de mortes maternas nas capitais brasileiras, ${ }^{7}$ as que detêm informações mais precisas, apontou para 2001 uma taxa sete vezes maior para as pretas comparadas às brancas e pardas.

A falta de conhecimento sobre quais fatores causam a disparidade na $\mathrm{MM}$ entre mulheres pretas e brancas 23 impede a formulação apropriada de intervenções para eliminar esta disparidade.

Em relação às disparidades raciais no Estado, os dados apontaram diferenças pouco significativas entre mulheres brancas e pardas acerca da RMM e $\mathrm{OR}$, no entanto, entre as pretas e indígenas, estes percentuais foram significativos, remetendo à necessidade de novas pesquisas para seu aprofundamento.

Por se tratar de estudo realizado com base em dados secundários, contou-se com as informações que se encontravam registradas nos bancos de dados utilizados. A falta de anotações de algumas informações nas fontes consultadas, como evidenciadas nas tabelas como dados ignorados, não favoreceu uma análise mais aprofundada, sendo uma limitação de estudos desta natureza.

Apesar do investimento do Ministério da Saúde em relação à saúde da mulher e introdução de novas equipes de saúde da família, a RMM - por causas evitáveis - não apresentou queda significativa em Mato Grosso. Houve incremento em termos quantitativos na cobertura pré-natal, mas os dados epidemiológicos não mostraram efetividade, necessitando investimentos na qualidade desse acompanhamento, bem como na redução de partos operatórios, pois, em 2006 na capital do Estado, houve $57,3 \%$ de partos operatórios pelo SUS, segundo o DATASUS, e aproximadamente $99 \%$ nos hospitais privados. 26

Elucidar as causas de disparidade racial relativas à mortalidade materna é essencial para que sejam elaboradas estratégias de redução para o grupo mais susceptível, bem como melhorar a assistência às afrodescendentes, consideradas as vulnerabilidades sociais a que estão expostas estas mulheres. 
2. Brasil. Ministério da Saúde. Secretaria de Políticas de Saúde. Área Técnica de Saúde da Mulher. Manual dos Comitês de Mortalidade materna. 3 ed. Brasília; 2007. (Série A. Normas e Manuais Técnicos).

3. WHO (World Health Organization). Mortality Country Fact Sheet 2006. [acesso em 5 nov 2009]. Disponível em http:// www.who.int/whosis/mort/profiles/mort_amro_bra_brazil. pdf

4. WHO (World Health Organization). Maternal mortality in 2005: estimates developed by WHO, UNICEF, UNFPA and the World Bank. Geneva; 2007. [acesso em 19 mar 2009]. Disponível em: http://www.who.int/whosis/mme_2005.pdf

5. Laurenti R, Jorge MHPM, Lebrão ML, Gotlieb SLD Estatísticas de Saúde. 2 ed. São Paulo: EPU; 2005.

6. Ayres JRCM, França Jr I, Calazans GJ, Saletti Filho HC Vulnerabilidade e prevenção em tempos de AIDS. In Barbosa RM, Parker R, org. Sexualidade pelo avesso: direitos, identidades e poder. São Paulo/Rio de Janeiro: Editora 34 IMS-UERJ; 1999. p. 49-72.

7. Batista LE, Escuder MML, Pereira JCR. A cor da morte: causas de óbito segundo características de raça no Estado de São Paulo, 1999 a 2001. Rev Saúde Pública. 2004; 38: 630-6.

8. Chor D, Lima CRA. Aspectos epidemiológicos das desigualdades raciais em saúde no Brasil. Cad Saúde Pública. 2005; 21: 1586-94

9. IBGE (Instituto Brasileiro de Geografia e Estatística). Censo demográfico e contagem da população. [acesso em 3 out 2009]. Disponível em: http://www.sidra.ibge.gov. $\mathrm{br} / \mathrm{bda} /$ tabela/protabl.asp $? \mathrm{c}=2093 \& \mathrm{z}=\mathrm{cd} \& \mathrm{o}=7 \& \mathrm{i}=\mathrm{P}$

10. Pinto E, Souzas R. A mortalidade materna e a questão raças/etnia: importância da lei do quesito cor no Sistema de Saúde. In: The Jojn and Catherine T. Perspectivas sobre saúde e direitos reprodutivos. São Paulo: MacArthur Foundation; 2002

11. Fang J, Madhavan S, Alderman MH. Maternal mortality in New York city: excess mortality of black women. J Urban Health. 2000; 77: 735-44.

12. Brasil. Ministério da Saúde. Secretaria de Atenção à Saúde. Departamento de Ações Programáticas Estratégicas. Estudo da mortalidade de mulheres de 10 a 49 anos, com ênfase na mortalidade materna. Brasília; 2006. (Série C. Projetos, Programas e Relatórios).

13. Rubin G. Reflexionando sobre el sexo: notas para una teoría radical de la sexualidad. In: Vance CS. Placer y peligro explorando la sexualidad femenina. Madrid: Ed. Revolución; 1989. p. 113-90

14. Razum O, Jahn A. Marital status and maternal mortality.
Lancet. 2000; 355: 2169.

15. Alexander GR, Kogan MD, Nabukera S. Racial differences in prenatal care use in the United states: arte disparities decreasing? Am J Public Health. 2002; 92: 1970-5.

16. Brasil. Ministério da Saúde. Secretaria de atenção à saúde. Departamento de ações programáticas estratégicas. Pacto nacional pela redução da mortalidade materna e neonatal. Brasília; 2004. [acesso em 8 set 2009]. Disponível em: http://dtr2002.saude.gov.br/proesf/Site/Arquivos_pdf_word /pdf/Pacto\%20Aprovado\%20na\%20Tripartite.pdf

17. Pereira WR. Poder, violência e dominação simbólicos em um serviço público de saúde que atende a mulheres em situação de gestação, parto e puerpério [tese]. Ribeirão Preto: Escola de Enfermagem de Ribeirão Preto da Universidade de São Paulo; 2000

18. Tanaka ACD. Mortalidade materna: reflexo da má qualidade e da desintegração dos serviços de saúde. J Rede Saúde. 2000; 20: 5-9.

19. Benevides MAS. Perspectiva da equidade no pacto nacional pela redução da mortalidade materna e neonatal: atenção à saúde das mulheres negras. Área Técnica de Saúde da Mulher / DAPE/SAS/MS. Brasília: Ministério da Saúde; 2007. (Série F. Comunicação e Educação em Saúde).

20. Fiscella K. Racial disparity in infant and maternal mortality: confluence of infection, and microvaascular dysfunction. Matern Child Health J. 2004; 8: 45-54.

21. Ronsmans C, Graham WJ. Maternal mortality: who, when, where, and why. Lancet. 2006; 368: 1189- 200.

22. Harper M, Dugan E, Espeland M, Martinez-Borges A, Mcquellon C. Why African-American women are at greater risk for pregnancy-related death. Ann Epidemiol. 2007; 17: 180-5.

23. Tucker MJ, Berg CJ, Callaghan WM, Hsia J. The blackwhite disparity in pregnancy-related mortality from 5 conditions: differences in prevalence and case-fatality rates. Am J Public Health. 2007; 97: 247-51

24. Adesse L, Monteiro MFG. Magnitude do aborto no Brasil: aspectos epidemiológicos e sócio-culturais. Brasília: Ipas Brasil / Instituto de Medicina Social; 2007. [acesso em 2 abr 2009]. Disponível em: http://www.ipas.org.br/arquivos/ factsh_mag.pdf

25. Martins AL. Mortalidade materna de mulheres negras no Brasil. Cad Saúde Pública. 2006; 22: 2473-9.

26. Brasil. Ministério da Saúde. DATASUS: informações de saúde. [acesso em 10 abr 2009]. Disponível em http://tabnet.datasus.gov.br/cgi/deftohtm.exe?sih/cnv/pimt. def

Recebido em 25 de março de 2011

Versão final apresentada em 29 de agosto de 2011

Aprovado em 1 de dezembro de 2011 